\title{
Finite-Difference Methods and the Eigenvalue Problem for Nonselfadjoint Sturm-Liouville Operators*
}

\section{By Alfred Carasso}

Abstract. In this paper we analyze the convergence of a centered finite-difference approximation to the nonselfadjoint Sturm-Liouville eigenvalue problem

$$
\begin{aligned}
& \Re[u] \equiv-\left[a(x) u^{\prime}\right]^{\prime}-b(x) u^{\prime}+c(x) u=\lambda u, \quad 0<x<1, \\
& u(0)=u(1)=0
\end{aligned}
$$

where $\mathfrak{Q}$ has smooth coefficients and $a(x) \geqq a_{0}>0$ on $[0,1]$. We show that the rate of convergence is $O\left(\Delta x^{2}\right)$ as in the selfadjoint case for a scheme of the same accuracy. We also establish discrete analogs of the Sturm oscillation and comparison theorems. As a corollary we obtain the result

$$
\lim _{M \rightarrow \infty ; \Delta_{x \rightarrow 0 ;}(M+1) \Delta_{x=1}}\left\{\sum_{p=1}^{M} \frac{\left\|V^{p}\right\|_{\infty}}{\Lambda_{p}}\right\}<\infty
$$

where $\Delta x=1 /(M+1)$ is the mesh size and $\Lambda_{p}, V^{p}$ are the characteristic pairs of $L$, the $M \times M$ matrix which approximates $\mathfrak{R}$, and $V^{p}$ is normalized so that $\left\|V^{p}\right\|_{2}=1$.

1. Introduction. Many authors (e.g. [1], [6], [8], [9]) have studied the convergence of finite-difference methods for selfadjoint Sturm-Liouville eigenvalue problems. In this report we are concerned with the nonselfadjoint problem

$$
\begin{aligned}
& \mathfrak{R}(u) \equiv-\left[a(x) u^{\prime}\right]^{\prime}-b(x) u^{\prime}+c(x) u=\lambda u, \quad 0<x<1, \\
& u(0)=u(1)=0
\end{aligned}
$$

where $a(x) \geqq a_{0}>0, c(x) \geqq 0$, and $b(x)$ are all smooth functions. This problem has an infinite sequence of positive [12, p. 37] and distinct [13, p. 212] eigenvalues

$$
0<\lambda_{1}<\lambda_{2}<\lambda_{3}<\cdots
$$

and a corresponding sequence of smooth eigenfunctions $u^{1}(x), u^{2}(x), u^{3}(x), \cdots$ which we assume normalized so that

$$
\int_{0}^{1}\left|u^{p}\right|^{2} d x=1, \quad p=1,2, \cdots .
$$

Of course, as is well known, the transformation

$$
u(x)=\left[\exp \left(-\frac{1}{2} \int_{0}^{x} \frac{b(t)}{a(t)} d t\right)\right] v(x)
$$

Received December 27, 1968, revised April 7, 1969.

* Supported by the Office of Naval Research under Contract No. N00014-67-A-1028-0004 at the University of Wisconsin. Reproduction in whole or in part is permitted for any purpose of the United States Government. 
puts (1.1) into the selfadjoint form

$$
\begin{aligned}
& \hat{L}[v] \equiv-\left(a v^{\prime}\right)^{\prime}+\left(c+\frac{1}{2} b^{\prime}+\frac{1}{4}\left(b^{2} / a\right) v=\lambda v,\right. \\
& v(0)=v(1)=0 .
\end{aligned}
$$

However, we consider the direct approximation of (1.1) by means of the finitedifference equations

$$
\begin{gathered}
-\frac{\left\{a_{k+1 / 2}\left(w_{k+1}-w_{k}\right)-a_{k-1 / 2}\left(w_{k}-w_{k-1}\right)\right\}}{\Delta x^{2}}-\frac{b_{k}\left(w_{k+1}-w_{k-1}\right)}{2 \Delta x} \\
+c_{k} w_{k}=\Lambda w_{k}, \quad k=1,2, \cdots, M, \\
w_{0}=w_{M+1}=0
\end{gathered}
$$

where $M$ is a large positive integer, $\Delta x=1 /(M+1)$ is the mesh spacing and the notation $g_{k}$ is used for $g(k \Delta x)$. Equivalently, we may write (1.5) as the finite-dimensional eigenvalue problem:

$$
L W=\Lambda W
$$

where $W$ is the $M$ component vector

$$
W=\left[\begin{array}{l}
w_{1} \\
w_{2} \\
\cdot \\
\cdot \\
\cdot \\
w_{M}
\end{array}\right]
$$

and $L$ the $M \times M$ tridiagonal matrix

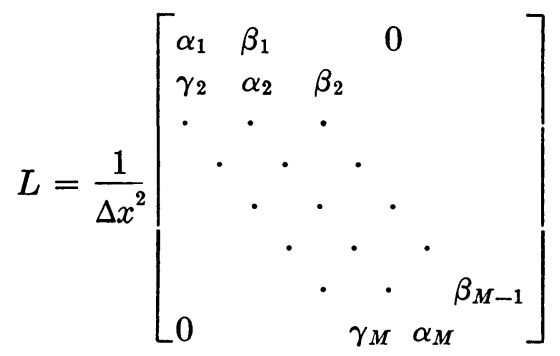

with

$$
\begin{aligned}
\alpha_{k} & =\left[a_{k+1 / 2}+a_{k-1 / 2}\right]+c_{k} \Delta x^{2} \beta_{k}=-\left[a_{k+1 / 2}+b_{k} \Delta x / 2\right] \quad \text { and } \\
\gamma_{k} & =\left[b_{k} \Delta x / 2-a_{k-1 / 2}\right] \quad k=1,2, \cdots, M .
\end{aligned}
$$

We will show that the latter procedure preserves the rate of convergence, namely $O\left(\Delta x^{2}\right)$, which obtains in the selfadjoint case for a scheme of the same accuracy, (see [6]). This is Theorem 1.

The matrix $L$ defined above will be shown to be similar to an oscillation matrix, by means of a diagonal transformation $\widetilde{D}$. Using the basic theorem on oscillation matrices, (see [4], [5]) and the fact that the entries of $\widetilde{D}$ alternate in sign, one immediately has a discrete analog of the Sturm Oscillation Theorem [13, p. 212, Theorem 
2.1] namely $L$ has positive distinct eigenvalues $0<\Lambda_{1}<\Lambda_{2}<\cdots<\Lambda_{M}$ and if $W^{j}$ is an eigenvector belonging to $\Lambda_{j}$ then $W^{j}$ has exactly $j-1$ nodes** in $0<x<1$. Moreover the nodes of successive eigenvectors alternate. ${ }^{* * *}$

We will also show the following

THEOREM 2. Let $V^{j}$ be an eigenvector of $L$ corresponding to $\Lambda_{j}$ and let $\delta_{\mathrm{Max}}\left(V^{j}\right)$ be the maximum distance between successive nodes of $V^{j}$. Then there exists an integer $j_{0}$, independent of $M$, and a positive constant $K_{1}$, such that for $j_{0} \leqq j \leqq M$, we have

$$
\delta_{\mathrm{Max}}\left(V^{j}\right) \leqq K_{1}\left(\Lambda_{j}\right)^{-1 / 2} .
$$

In the continuous case, the estimate (1.9) is usually obtained as a corollary to the Sturm Comparison Theorem [14, p. 224]. We will base the proof of Theorem 2 on a discrete maximum principle. We remark that in the continuous case, proofs of the oscillation and comparison theorems, based on a maximum principle, have been given by Protter and Weinberger in their recent book. See [12].

\section{Symmetrization of the Discrete Problem.}

Definitions. For any two $M$ vectors $X, Y$ define their scalar products by

$$
\langle X, Y\rangle=\Delta x \sum_{k=1}^{M} x_{k} \bar{y}_{k}
$$

and let

$$
\|X\|_{2}=\left\{\Delta x \sum_{k=1}^{M}\left|x_{k}\right|^{2}\right\}^{1 / 2}
$$

be the corresponding norm.

If $A$ is an $M \times M$ matrix then we define

$$
\|A\|_{2}=\operatorname{Sup}_{X \neq 0} \frac{\|A X\|_{2}}{\|X\|_{2}}
$$

Lemma 1. Fix $\Delta x>0$ sufficiently small. Then there exists a nonsingular, positive, diagonal matrix $D$ such that $D^{-1} L D=\hat{L}$ is a real symmetric matrix. Moreover, $\|D\|_{2}$, $\left\|D^{-1}\right\|_{2}$ remain bounded as $M \rightarrow \infty, \Delta x \rightarrow 0,(M+1) \Delta x=1$.

Proof. We construct such a matrix. Let

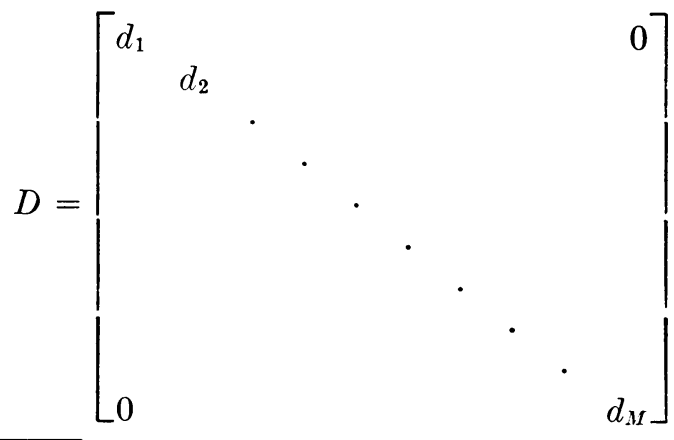

where $d_{j} \neq 0, j=1, \cdots, M$

** As in [5], a "node" of $V^{i}$ is a point where the graph of $V^{i}$, (i.e. the graph of the piecewiselinear function obtained from $V^{i}$ by linear interpolation) intersects the $x$-axis.

*** These observations about $L$ are not new. (See Sinden [17] and Varga [18, p. 206].) 
and $d_{1}=1$ and let $\hat{L}=D^{-1} L D=\left(l_{i j}\right)$.

Since we require $\hat{L}=\hat{L}^{T}$ we must have

$$
d_{i}^{-1} l_{i j} d_{j}=d_{j}^{-1} l_{j i} d_{i} \quad \text { where } L=\left(l_{i j}\right) .
$$

Further, since $l_{i j}=0$ for $j>i+1, j<i-1$, the $d_{j}$ 's must be determined so that

$$
d_{i}{ }^{2}=\frac{l_{i, i-1}}{l_{i-1, i}} d_{i-1}^{2}, \quad i=2, \cdots, M .
$$

Starting from $d_{1}=1$, we may solve recursively to obtain

$$
d_{i}{ }^{2}=\prod_{k=1}^{i-1}\left(\frac{\gamma_{k+1}}{\beta_{k}}\right), \quad i=2, \cdots, M
$$

and, since $\gamma_{k}, \beta_{k}<0$ for sufficiently small $\Delta x, d_{i}{ }^{2}>0$ if $\Delta x$ is small enough.

With $D$ constructed as above, we have

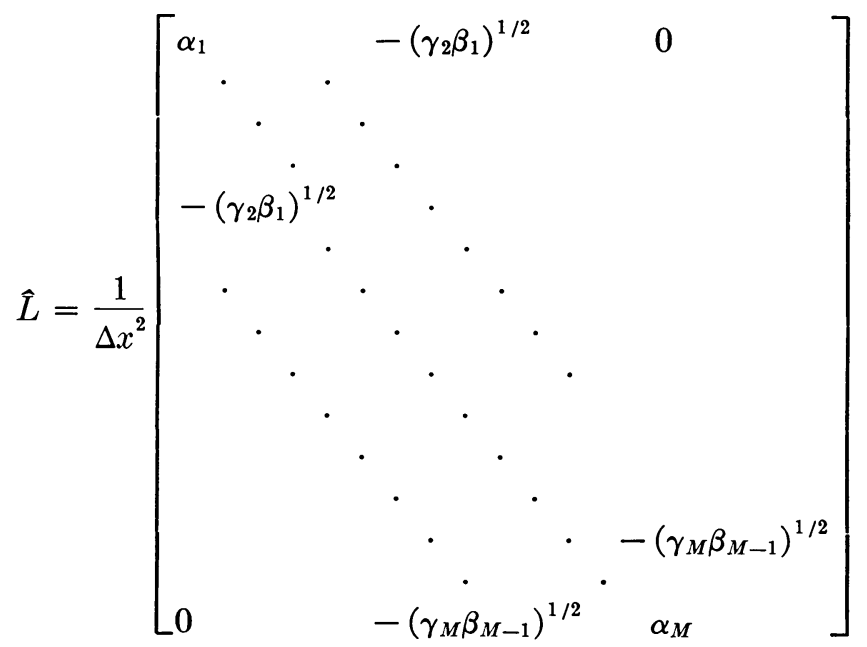

and we must show that $\|D\|_{2},\left\|D^{-1}\right\|_{2}$ remain bounded as $M \rightarrow \infty$. Let

$$
Q_{i}=\prod_{k=1}^{i-1}\left(1-\frac{b_{k+1} \Delta x}{2 a_{k+1 / 2}}\right)
$$

and

$$
P_{i}=\prod_{k=1}^{i-1}\left(1+\frac{b_{k} \Delta x}{2 a_{k+1 / 2}}\right)
$$

then $d_{i}{ }^{2}=Q_{i} / P_{i}$. Now for sufficiently small $\Delta x$,

$$
\log \left(1-\frac{b_{k+1} \Delta x}{2 a_{k+1 / 2}}\right)=-\frac{b_{k+1} \Delta x}{2 a_{k+1 / 2}}+O\left(\Delta x^{2}\right)
$$

so that

$$
\log Q_{i}=-\Delta x \sum_{k=1}^{i-1} \frac{b_{k}}{2 a_{k+1 / 2}}+\Delta x \sum_{k=1}^{i-1} O(\Delta x)
$$


Hence,

$$
\lim _{\Delta x \rightarrow 0, i \rightarrow \infty ; i \Delta x=\bar{x}}\left[\log Q_{i}\right]=-\frac{1}{2} \int_{0}^{\bar{x}} \frac{b(t)}{a(t)} d t .
$$

Similarly,

$$
\lim _{\Delta x \rightarrow 0, i \rightarrow \infty ; i \Delta x=x}\left[\log P_{i}\right]=\frac{1}{2} \int_{0}^{\bar{x}} \frac{b(t)}{a(t)} d t .
$$

Consequently,

$$
\lim d_{i}=\left[\exp \left(-\frac{1}{2} \int_{0}^{\bar{x}} \frac{b(t)}{a(t)} d t\right)\right] \leqq K_{0}<\infty
$$

which shows both $\|D\|_{2},\left\|D^{-1}\right\|_{2}$ remain bounded as $\Delta x \rightarrow 0, M \rightarrow \infty,(M+1) \Delta x$ $=1$.

Lemma 2. For $\Delta x$ sufficiently small, the eigenvalues of $L$ are strictly positive and they remain bounded away from zero as $M \rightarrow \infty, \Delta x \rightarrow 0,(M+1) \Delta x=1$.

Proof. For $\Delta x$ sufficiently small, $\gamma_{k}, \beta_{k}<0$. Hence if $L=\left(l_{i j}\right)$ and $\Omega_{i}=$ $\sum_{j \neq i}\left|l_{i j}\right|$, then

$$
\Omega_{i}=\left(a_{i+1 / 2}+a_{i-1 / 2}\right) / \Delta x^{2}
$$

and $l_{i i}=\left(a_{i+1 / 2}+a_{i-1 / 2}\right) / \Delta x^{2}+c_{i} \geqq \Omega_{i}$ since $c_{i} \geqq 0$.

By Gershgorin's theorem, [7], the eigenvalues of $L$ lie in the union of the discs $\left|z-l_{i i}\right| \leqq \Omega_{i}$ in the complex plane. Hence if $\Lambda$ is an eigenvalue of $L$, then $\Lambda \geqq 0$ since $\Lambda$ is real.

Now let $l_{h}$ be the finite-difference operator corresponding to $-L$, i.e.

$$
\begin{aligned}
{\left[l_{h} v\right]_{k} \equiv } & -\left[\frac{\left(a_{k+1 / 2}+a_{k-1 / 2}\right)+c_{k} \Delta x^{2}}{\Delta x^{2}}\right] v_{k}+\left[\frac{a_{k+1 / 2}+b_{k} \Delta x / 2}{\Delta x^{2}}\right] v_{k+1} \\
& +\left[\frac{a_{k-1 / 2}-b_{k} \Delta x / 2}{\Delta x^{2}}\right] v_{k-1} .
\end{aligned}
$$

Then, for sufficiently small $\Delta x, l_{h}$ is of positive type [3, p. 181] and so satisfies the discrete maximum principle [16, p. 23, Lemma 2.3]. Consequently [16, p. 108, Theorem $7.1]$ if $w(k \Delta x), k=0,1, \cdots, M+1$ is an arbitrary real-valued mesh function, there exists positive constants $K$ and $\delta$ such that if $0<\Delta x<\delta$,

$$
\|w\|_{\infty} \equiv \operatorname{Max}_{k}\left|w_{k}\right| \leqq \operatorname{Max}\left\{\left|w_{0}\right|,\left|w_{M+1}\right|\right\}+K\left\|\left(l_{h} w\right)\right\|_{\infty} .
$$

Now let $V=\left\{v_{k}\right\}_{k=1}^{M}$ be an eigenvector of $L$ corresponding to $\Lambda$. We may assume $V$ to be real. Defining $v_{0}=v_{M+1}=0, L V=\Lambda V$ is equivalent to

$$
\left[l_{h} v\right]_{k}=-\Lambda v_{k}, \quad k=1, \cdots, M .
$$

Hence, using (2.2) and the fact that $\Lambda \geqq 0$,

$$
\|v\|_{\infty} \leqq K\left\|\left(l_{h} v\right)\right\|_{\infty}=\Lambda K\|v\|_{\infty}
$$

i.e. $\Lambda \geqq 1 / K>0$. Q.E.D.

Corollary. Let $\Gamma$ be the $M \times M$ matrix given by 


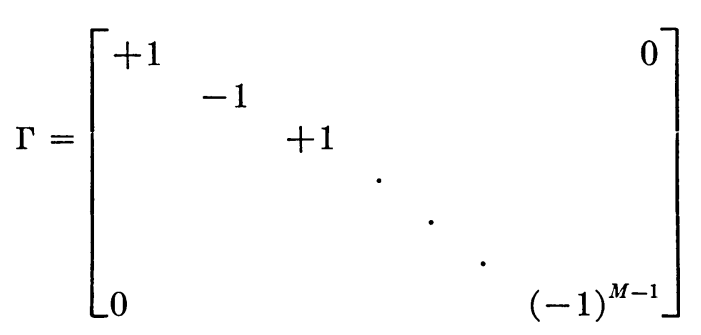

and let $\hat{L}$ be defined by (2.1), then $\Gamma^{-1} \hat{L} \Gamma$ is an oscillation matrix.

Proof. $\Gamma^{-1} \hat{L} \Gamma$ is a positive-definite real symmetric matrix with positive elements along the first super and sub diagonals. The proof now follows from a theorem of Gantmacher and Krein [4, p. 103].

3. Convergence of the Characteristic Pairs of $L$. Let $0<\Lambda_{1}<\Lambda_{2}<\cdots<\Lambda_{M}$ be the eigenvalues of $L$. Fix a positive integer $p$ and let $V^{p}(\Delta x)$ be the eigenvector corresponding to $\Lambda_{p}(\Delta x)$, normalized so that $\left\|V^{p}\right\|_{2}=1$. Let $\tilde{V}^{p}$ be the continuous piecewise-linear function, vanishing at $x=0,1$, and which, in the interior of $[0,1]$, is obtained from $V^{p}$ by linear interpolation. Consider the families $\left\{\Lambda_{p}(\Delta x)\right\}$, $\left\{\tilde{V}^{p}(\Delta x)\right\}$ as the mesh size $\Delta x \rightarrow 0$.

Using Lemma 1 and considering the symmetrized problem, one can give a direct proof of uniform convergence of $\widetilde{V}^{p}$ to $u^{p}$ and $\Lambda_{p}$ to $\lambda_{p}$ as $\Delta x \rightarrow 0$. (See [2].) This method of proof is based on the compactness of the family $\left\{\widetilde{V}^{p}(\Delta x)\right\}$ in $C[0,1]$ and has been used several times by Parter (see [9], [10], [11]) but it does not immediately yield estimates on the rates of convergence. Nevertheless we will make use below (see Eq. 3.8 (1)) of the fact that $\Lambda_{p} \rightarrow \lambda_{p}$ together with Lemma 1 above to obtain these estimates. The proof given below is a modification of that given by Gary in [6] for the selfadjoint case.

Theorem 1. Let $\Lambda_{p}, V^{p}$ be characteristic pairs of $L$ with $\left\|V^{p}\right\|_{2}=1$. Let $D$ be the diagonal matrix of Lemma 1. Let $u^{p}$ be an eigenfunction of $\&$ corresponding to $\lambda_{p}$ and let $U^{p}$ be the $M$ vector obtained from $u^{p}$ by mesh-point evaluation. Assume $u^{p}(x)$ normalized so that

$$
\left\|D^{-1} U^{p}\right\|_{2}=\left\|D^{-1} V^{p}\right\|_{2}
$$

then as $\Delta x \rightarrow 0$, we have

$$
\begin{aligned}
\left|\lambda_{p}-\Lambda_{p}\right| & \leqq K \Delta x^{2}, \\
\left\|U^{p}-V^{p}\right\|_{2} & \leqq K_{1} \Delta x^{2}
\end{aligned}
$$

where $K, K_{1}$ are positive constants depending only on $p$.

Proof. Because the difference scheme in (1.5) is properly centered and we assume sufficient smoothness of $u^{p}$ and the coefficients of $\mathfrak{Z}$, we have at the mesh points,

$$
\mathfrak{R}\left[u^{p}\right]=L U^{p}+\tau=\lambda_{p} U^{p}
$$

where $\tau$ is the "truncation" error and

$$
\|\tau\|_{2} \leqq K(p) \Delta x^{2} \quad \text { where } K \text { is a constant . }
$$

Let $\hat{L}=D^{-1} L D$ have orthonormal eigenvectors $X^{1}, X^{2}, \cdots, X^{M}$ and write $U^{p}$ as a linear combination of the $D X^{j}$ 's: 


$$
U^{p}=\sum_{j=1}^{M} \sigma_{j} D X^{j}
$$

so that

$$
L U^{p}=\sum_{j=1}^{M} \sigma_{j} L D X^{j}=\sum_{j=1}^{M} \sigma_{j} \Lambda_{j} D X^{j}
$$

then

$$
\tau=\left(\lambda_{p}-L\right) U^{p}=\sum_{j=1}^{M} \sigma_{j}\left(\lambda_{p}-\Lambda_{j}\right) D X^{j}
$$

and

$$
\sum_{j=1}^{M}{\sigma_{j}}^{2}\left|\lambda_{p}-\Lambda_{j}\right|^{2}=\left\|D^{-1} \tau\right\|_{2}{ }^{2} \leqq\left\|D^{-1}\right\|_{2}{ }^{2}\|\tau\|_{2}{ }^{2} \leqq K_{1}(p) \Delta x^{4}
$$

where $K_{1}$ is a constant.

Now, the eigenvalues of $L$ are distinct and converge to the corresponding distinct eigenvalues of $\mathfrak{R}$. It follows that

$$
\inf _{j \neq p}\left\{\left|\lambda_{p}-\Lambda_{j}\right|\right\} \geqq \omega_{0}>0
$$

for all sufficiently small $\Delta x$. Hence, on using (3.7),

$$
\sum_{j \neq p}{\sigma_{j}}^{2} \leqq K_{1} \Delta x^{4}
$$

From (3.9), (3.6) we obtain

$$
{\sigma_{p}}^{2}=\left\|D^{-1} U^{p}\right\|_{2}{ }^{2}+O\left(\Delta x^{4}\right) \geqq \omega_{1}>0
$$

for all sufficiently small $\Delta x$.

Thus

$$
\left|\lambda_{p}-\Lambda_{p}\right| \leqq K_{2}(p) \Delta x^{2}
$$

Since $V^{p}=\beta D X^{p}$ for some $\beta$ and $\left\|X^{p}\right\|_{2}=1$ we have

$$
|\beta|=\left\|D^{-1} V^{p}\right\|_{2} \text {. }
$$

On taking square roots in (3.10), we have

$$
\sigma_{p}=\left\|D^{-1} U^{p}\right\|_{2}+O\left(\Delta x^{4}\right)
$$

and we may assume that $\sigma_{p}$ and $\beta$ have the same sign; hence using (3.1),

$$
\left(\sigma_{p}-\beta\right)=O\left(\Delta x^{4}\right) \text {. }
$$

Writing $U^{p}-V^{p}=\sum_{j_{\neq p}^{p}} \sigma_{j} D X^{j}+\left(\sigma_{p}-\beta\right) D X^{p}$ we have

$$
\left\|D^{-1}\left(U^{p}-V^{p}\right)\right\|_{2}^{2}=\sum_{j \neq p}{\sigma_{j}}^{2}+\left(\sigma_{p}-\beta\right)^{2}=O\left(\Delta x^{4}\right)
$$

i.e.

$$
\left\|U^{p}-V^{p}\right\|_{2}^{2} \leqq\|D\|_{2}{ }^{2}\left\|D^{-1}\left(U^{p}-V^{p}\right)\right\|_{2}^{2} \leqq K_{3}(p) \Delta x^{4} \text {. Q.E.D. }
$$

Notice that the above inequality also implies uniform convergence at the rate of $O(\Delta x)^{3 / 2}$. 


\section{Proof of Theorem 2.}

Lemma 3. Let $0<\Lambda_{1}<\cdots<\Lambda_{M}$ be the eigenvalues of $L$. Then there exists a positive integer $j_{0}$, independent of $M$, such that for $j_{0} \leqq j \leqq M$ we have

$$
K_{1} j^{2} \pi^{2} \leqq \Lambda_{j} \leqq K_{2} j^{2} \pi^{2}, \quad K_{1}, K_{2} \text { positive constants } .
$$

Proof. In the selfadjoint case this result may be found in Bückner [1]. In the present more general case we will need to estimate the off-diagonal elements of the matrix $\hat{L}$ in Lemma 1 .

With the notation of (1.8) let

$$
q_{k}^{2}=\gamma_{k+1} \beta_{k}=\left(a_{k+1 / 2}-\frac{b_{k+1} \Delta x}{2}\right)\left(a_{k+1 / 2}+\frac{b_{k} \Delta x}{2}\right), \quad k=1, \cdots, M-1 .
$$

Since $b(x) \in C^{1}[0,1]$, we have by the mean-value theorem,

$$
q_{k}^{2}=\left(a_{k+1 / 2}\right)^{2}\left[1-2 \mu_{k} \Delta x^{2}+O\left(\Delta x^{3}\right)\right]
$$

where $2 \mu_{k}=\left[b_{k}{ }^{2}+2 a_{k+1 / 2} b^{\prime}\left(\xi_{k}\right)\right] / 4 a_{k+1 / 2}$ for some $\xi_{k}$ such that $k \Delta x<\xi_{k}<(k+1) \Delta x$. Hence on taking square roots

$$
q_{k}=a_{k+1 / 2}\left[1-\mu_{k} \Delta x^{2}+O\left(\Delta x^{3}\right)\right], \quad k=1, \cdots, M-1 .
$$

We now proceed to estimate the quadratic form $\langle X, \hat{L} X\rangle$ where $X$ is any complex $M$ vector of norm 1. Defining $x_{0}=x_{M+1}=0$, and using (4.3), we may write

$$
\begin{aligned}
\langle X, \hat{L} X\rangle= & \Delta x \sum_{k=0}^{M} \frac{\left|x_{k}-x_{k+1}\right|^{2}}{\Delta x^{2}}+\Delta x \sum_{k=1}^{M} c_{k}\left|x_{k}\right|^{2} \\
& +2 \Delta x \sum_{k=0}^{M} \mu_{k} a_{k+1 / 2} x_{k} \bar{x}_{k+1}+O(\Delta x) \Delta x \sum_{k=0}^{M} x_{k} \bar{x}_{k+1} .
\end{aligned}
$$

Now let $0<a_{0} \leqq a(x) \leqq a_{1}$ on $[0,1]$ and let

$$
\|c\|_{\infty}=\operatorname{Max}_{k}\left|c_{k}\right|, \quad\|\mu\|_{\infty}=\operatorname{Max}_{k}\left|\mu_{k}\right| \text {. }
$$

We have

$$
\langle X, \hat{L} X\rangle \leqq a_{1} \Delta x \sum_{k=0}^{M} \frac{\left|x_{k+1}-x_{k}\right|^{2}}{\Delta x^{2}}+\|c\|_{\infty}+2 a_{1}\|\mu\|_{\infty}+|O(\Delta x)|
$$

and

$$
\langle X, \hat{L} X\rangle \geqq a_{0} \Delta x \sum_{k=0}^{M} \frac{\left|x_{k+1}-x_{k}\right|^{2}}{\Delta x^{2}}-2 a_{1}\|\mu\|_{\infty}-|O(\Delta x)| .
$$

Let $H$ be the tridiagonal $M \times M$ matrix defined by

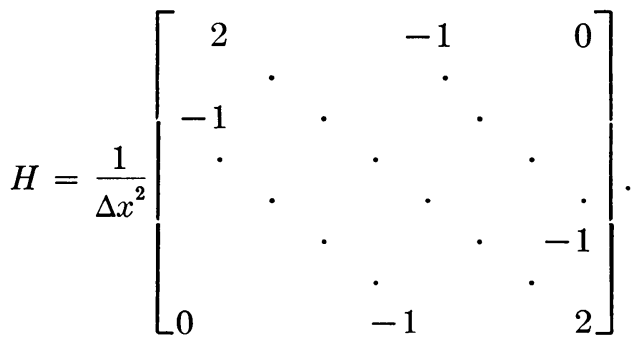


It is easily verified that

$$
\langle X, H X\rangle=\Delta x \sum_{k=0}^{M} \frac{\left|x_{k+1}-x_{k}\right|^{2}}{\Delta x^{2}}
$$

and that the eigenvalues $\theta_{j}, j=1, \cdots, M$, of $H$, arranged in increasing order, are given by

$$
\theta_{j}=\frac{4}{\Delta x^{2}} \sin ^{2} \frac{j \pi \Delta x}{2}, \quad j=1, \cdots, M .
$$

Inserting (4.9) into (4.6), (4.7) and using the maximum principle for the eigenvalues of real symmetric matrices shows that

(4.11) $\quad a_{0} \theta_{j}-2\|\mu\|_{\infty}-|O(\Delta x)| \leqq \Lambda_{j} \leqq a_{1} \theta_{j}+\|c\|_{\infty}+2 a_{1}\|\mu\|_{\infty}+|O(\Delta x)|$.

Using (4.10) and an elementary calculation, the proof follows from (4.11).

Proof of Theorem 2. Let

$$
W^{j}=\left[\begin{array}{l}
w_{1}{ }^{j} \\
\cdot \\
\cdot \\
\cdot \\
w_{M}{ }^{j}
\end{array}\right]
$$

be an eigenvector of $L$ corresponding to $\Lambda_{j}$. Then $W^{j}$ satisfies the difference equations:

$$
\begin{aligned}
-\left[2+\frac{\left(c_{k}-\Lambda_{j}\right) \Delta x^{2}}{\omega_{k}}\right] w_{k}^{j} & +\left[\frac{a_{k+1 / 2}+b_{k} \Delta x / 2}{\omega_{k}}\right] w_{h+1}^{j} \\
+ & {\left[\frac{a_{k-1 / 2}-b_{k} \Delta x / 2}{\omega_{k}}\right] w_{k-1}^{j}=0, \quad k=1, \cdots, M }
\end{aligned}
$$

where $w_{0}^{j}=w_{M+1}^{j}=0$ and $\omega_{k}=\frac{1}{2}\left(a_{k+1 / 2}+a_{k-1 / 2}\right)$.

Let

$$
\begin{aligned}
& \tilde{\alpha}_{k}=-\left[2+\frac{\left(c_{k}-\frac{\Lambda_{j}}{\omega_{k}}\right) \Delta x^{2}}{\omega_{k}}\right], \quad \tilde{\beta}_{k}=\left[\frac{a_{k+1 / 2}+\frac{1}{2} b_{k} \Delta x}{\omega_{k}}\right], \\
& \tilde{\gamma}_{k}=\left[\frac{a_{k-1 / 2}-\frac{1}{2} b_{k} \Delta x}{\omega_{k}}\right],
\end{aligned}
$$

and let $A$ be the tridiagonal $M \times M$ matrix

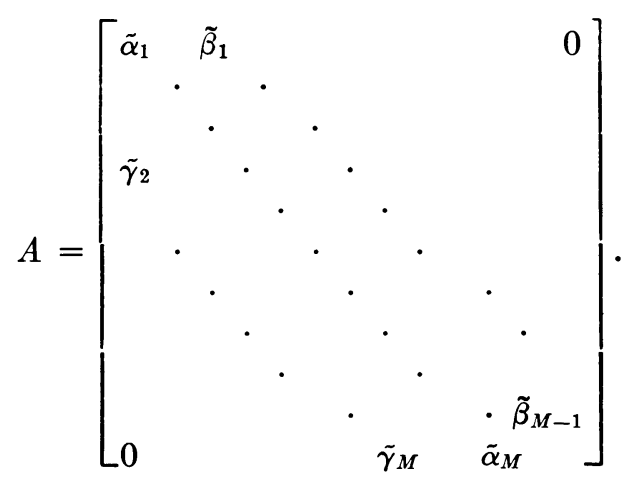


Then we may write (4.12) as

$$
A W^{j}=0
$$

or equivalently

$$
\left(P^{-1} A P\right) P^{-1} W^{j}=0
$$

if $P$ is any nonsingular matrix.

Choose $P$ to be the diagonal matrix

$$
P=\left[\begin{array}{rrrrrrr}
p_{1} & & & & & & 0 \\
& \cdot & & & & & \\
& & \cdot & & & & \\
& & & \cdot & & & \\
& & & & \cdot & \\
0 & & & & & p_{M}
\end{array}\right]
$$

where $p_{1}=1$ and $p_{i}{ }^{2}=\prod_{k=1}^{i-1}\left(\tilde{\gamma}_{k+1} / \tilde{\beta}_{k}\right), i=2, \cdots, M$.

For all sufficiently small $\Delta x, p_{i}{ }^{2}>0$ and as in Lemma $1, P$ symmetrizes $A$. Let $\sigma_{k}=\left(\tilde{\gamma}_{k+1} \tilde{\beta}_{k}\right)^{1 / 2}$, then

$$
P^{-1} A P=\left[\begin{array}{cccccccc}
\tilde{\alpha}_{1} & & \sigma_{1} & & & & & 0 \\
& \cdot & & \cdot & & & \\
\sigma_{1} & & \cdot & & \cdot & & \\
& \cdot & & \cdot & & \cdot & \\
& & \cdot & & \cdot & & \sigma_{M-1} \\
& & & \cdot & & \cdot & \\
0 & & & & \sigma_{M-1} & \tilde{\alpha}_{M}
\end{array}\right] .
$$

Observe that by the mean-value theorem

$$
\omega_{k} \omega_{k+1}=\left(a_{k+1 / 2}\right)^{2}\left[1+O\left(\Delta x^{2}\right)\right] \quad \text { as } \Delta x \rightarrow 0 .
$$

Also if $b(x) \in C^{1}[0,1]$,

$$
\begin{aligned}
\left(\tilde{\gamma}_{k+1} \tilde{\beta}_{k}\right) & =\left[\frac{\left.\left(a_{k+1 / 2}\right)^{2}+\frac{a_{k+1 / 2}\left(b_{k}-b_{k+1}\right) \Delta x}{2}-\frac{b_{k} b_{k+1} \Delta x^{2}}{4}\right]}{\omega_{k} \omega_{k+1}}\right] \\
& =\frac{\left(a_{k+1 / 2}\right)^{2}\left[1+O\left(\Delta x^{2}\right)\right] \quad \text { as } \Delta x \rightarrow 0 .}{\left(a_{k+1 / 2}\right)^{2}\left[1+O\left(\Delta x^{2}\right)\right] \quad} \quad .
\end{aligned}
$$

Hence,

$$
\sigma_{k}=\left(\tilde{\gamma}_{k+1} \tilde{\beta}_{k}\right)^{1 / 2}=1+O\left(\Delta x^{2}\right) \quad \text { as } \Delta x \rightarrow 0 .
$$

Let $V=P^{-1} W^{j}$ and write the system (4.15) as

$$
\begin{aligned}
-\left[2+\frac{\left(c_{k}-\Lambda_{j}\right) \Delta x^{2}}{\omega_{k}}\right] v_{k}+\sigma_{k} v_{k+1}+\sigma_{k} v_{k-1} & =0, \\
v_{0}=v_{M+1} & =0, \quad k=1, \cdots, M .
\end{aligned}
$$

Let $K_{1}$ and $K_{2}$ be the constants in Lemma 3 and define 


$$
\beta_{j}{ }^{2}=\Lambda_{j} / K_{2} .
$$

Let $y(x)=\sin \beta_{j} x$. Then $y_{k}=y(k \Delta x)$ satisfies the difference equations:

$$
-\left[2-\mu_{j} \Delta x^{2}\right] y_{k}+y_{k+1}+y_{k-1}=0, \quad k=1,2, \cdots
$$

where

$$
\mu_{j}=\frac{4}{\Delta x^{2}} \sin ^{2} \frac{\beta_{j} \Delta x}{2} .
$$

The distance between successive zeros of $y(x)$ is $\pi / \beta_{j}=\left(K_{2} \pi^{2} / \Lambda_{j}\right)^{1 / 2} \geqq 1 / j$ for $j$ large enough by Lemma 3 .

Let $v(x)$ be the piecewise-linear function corresponding to "graph" of vector $V=P^{-1} W^{j}$. Define the auxiliary function $z(x)$ by

$$
z(x)=y(x) / v(x) \quad \text { whenever } v(x) \neq 0 .
$$

We proceed to estimate the distance between successive nodes of $v(x)$ by investigating the difference equation satisfied by $z(x)$.

We may assume that $\delta_{\operatorname{Max}}(V)>3 \Delta x$; for if $\delta_{\operatorname{Max}}(V) \leqq 3 \Delta x$, then in particular, $\delta_{\operatorname{Max}}(V) \leqq 3 /(M+1)<3 / j \leqq 3 \pi\left(K_{2} / \Lambda_{j}\right)^{1 / 2}$ for all sufficiently large $j$. If $\delta_{\operatorname{Max}}>3 \Delta x$, then there exists a set $N$ of consecutive mesh points, containing at least three members on which $v(x)$ is strictly positive (or strictly negative). Let $N^{\prime}$ be $N$ minus the two end points of $N$. Since $z_{k}=y_{k} / v_{k}$ for $k \in N^{\prime}$,

$$
\begin{aligned}
{\left[l_{h}\right]_{k} \equiv } & -\left[\frac{\left(2-\mu_{j} \Delta x^{2}\right) \sigma_{k}}{2+\left(c_{k}-\Lambda_{j}\right) \Delta x^{2} / \omega_{k}}\left(v_{k+1}+v_{k-1}\right)\right] z_{k} \\
& +v_{k+1} z_{k+1}+v_{k-1} z_{k-1}=0, \quad k \in N^{\prime} .
\end{aligned}
$$

We now show that for all sufficiently large $j$, the difference operator $l_{h}$ (or $-l_{h}$ if $v$ is strictly negative) occurring in (4.25) is of positive type, and hence satisfies the discrete maximum principle:

It is sufficient to show that if $j$ is sufficiently large,

$$
\frac{\left[2-\mu_{j} \Delta x^{2}\right] \sigma_{k}}{2+\left(c_{k}-\Lambda_{j}\right) \Delta x^{2} / \omega_{k}} \geqq 1, \quad \text { if } k \in N^{\prime} .
$$

From (4.24) we have $\mu_{j} \leqq \Lambda_{j} / K_{2} \leqq \Lambda_{j} / 2 a_{1}$ if $K_{2}$ is chosen so that $K_{2} \geqq 2 a_{1}$, where $a_{1}$ is an upper bound for $a(x)$ on $[0,1]$. Hence,

$$
\left(2-\mu_{j} \Delta x^{2}\right) \sigma_{k}=2-\mu_{j} \Delta x^{2}+O\left(\Delta x^{2}\right)
$$

since $\mu_{j} \Delta x^{2} \leqq 4$ and $\sigma_{k}=1+O\left(\Delta x^{2}\right)$. Now,

$$
\begin{aligned}
2-\mu_{j} \Delta x^{2}+O\left(\Delta x^{2}\right) & \geqq 2-\Lambda_{j} \Delta x^{2} / K_{2}+O\left(\Delta x^{2}\right) \\
& \geqq 2-\Lambda_{j} \Delta x^{2} / 2 \omega_{k}+O\left(\Delta x^{2}\right) \\
& =2+\frac{\left(c_{k}-\Lambda_{j}\right) \Delta x^{2}}{\omega_{k}}+\frac{\left(\Lambda_{j}-2 c_{k}\right) \Delta x^{2}}{2 \omega_{k}}+O\left(\Delta x^{2}\right)
\end{aligned}
$$

i.e.

$$
\left(2-\mu_{j} \Delta x^{2}\right) \sigma_{k} \geqq 2+\left(c_{k}-\Lambda_{j}\right) \Delta x^{2} / \omega_{k}
$$


if $j$ is sufficiently large, since we assume $c(x)$ is bounded.

Furthermore, $2+\left(c_{k}-\Lambda_{j}\right) \Delta x^{2} / \omega_{k}$ is positive for $k \in N^{\prime}$ since $v_{k}, v_{k+1}, v_{k-1}$ have the same sign, on using (4.21). Thus (4.26) is satisfied.

Suppose now that $z(x)$ has two zeros in the interval spanned by $N$. At any mesh point lying between the two zeros we must have $z(x)=0$ by the maximum principle. Since $z(x)=0$ if and only if $y(x)=0$, this means that the distance between successive zeros of $y(x)$ is $\leqq \Delta x=1 /(M+1)$. However, as already noted, this distance is $\geqq 1 / j$ and $j \leqq M$.

Thus $y(x)$ has at most one zero in the interval spanned by $N$. Hence the maximum distance between successive nodes of $v(x)$ must be less than or equal to $\pi / \beta_{j}$ $+2 \Delta x$. Since $\Lambda_{j}=O\left(1 / \Delta x^{2}\right)$, we have

$$
\delta_{\mathrm{Max}}(V) \leqq K\left(\Lambda_{j}\right)^{-1 / 2} .
$$

A similar estimate is valid for the eigenvector $W^{j}$ of $L$ since $W^{j}=P V$ and $P$ is a positive diagonal matrix. Q.E.D.

Corollary 1. Let the eigenvectors $\left\{V^{p}\right\}$ of $L$ be normalized so that $\left\|V^{p}\right\|_{2}=1$. Then there exists a constant $K$ and an integer $p_{0}$, both independent of $M$ such that if $p_{0} \leqq p \leqq M$

$$
\left\|V^{p}\right\|_{\infty} \equiv \operatorname{Max}_{k=1 \cdots M}\left|v_{k}^{p}\right| \leqq K p^{1 / 2} .
$$

Proof. Let $W^{p}$ be the normalized eigenvector of $\hat{L}=D^{-1} L D$ corresponding to $\Lambda_{p}$. Since $W^{p}=D^{-1} V^{p} /\left\|D^{-1} V^{p}\right\|_{2}$ and $D^{-1}$ is a positive diagonal matrix, the distance between successive nodes of $W^{p}$ satisfies an estimate similar to (4.29). Since $W^{p}$ is normalized we have

$$
\left\langle W^{p}, \hat{L} W^{p}\right\rangle=\Lambda_{p} \text {. }
$$

Hence, using inequality (4.7) in the proof of Lemma 3, we get,

$$
\Delta x \sum_{k=0}^{M} \frac{\left|w_{k+1}^{p}-w_{k}^{p}\right|^{2}}{\Delta x^{2}} \leqq \frac{2 \Lambda_{p}}{a_{0}}
$$

for all sufficiently large $p$.

Let $r, s$ be any two positive integers with $1 \leqq s<r \leqq M$. Then,

$$
\begin{aligned}
\left|w_{r}^{p}-w_{s}^{p}\right| & =\left|\Delta x \sum_{k=s}^{r-1} \frac{w_{k+1}^{p}-w_{k}^{p}}{\Delta x}\right| \\
& \leqq[(r-s) \Delta x]^{1 / 2}\left(\Delta x \sum_{k=0}^{M} \frac{\left|w_{k+1}^{p}-w_{k}^{p}\right|^{2}}{\Delta x^{2}}\right)^{1 / 2} \\
& \leqq[(r-s) \Delta x]^{1 / 2}\left(2 \Lambda_{p} / a_{0}\right)^{1 / 2}
\end{aligned}
$$

on using Schwarz's inequality and (4.32). Now choose $r$ so that $\left|w_{r}^{p}\right|=\left\|W^{p}\right\|_{\infty}>0$ and let $s$ be the integer nearest $r$ with the property that $w_{s}{ }^{p} w_{r}{ }^{p} \leqq 0$. ( $s$ need not necessarily be less than $r$.) We then have for sufficiently large $p$, by Theorem 2 ,

$$
|(r-s) \Delta x|<2 \delta_{\mathrm{Max}}\left(W^{p}\right) \leqq K^{\prime}\left(\Lambda_{p}\right)^{-1 / 2} .
$$

Hence using (4.33), (4.34) 


$$
\begin{aligned}
\left\|W^{p}\right\|_{\infty} & \leqq\left|w_{r}{ }^{p}-w_{s}{ }^{p}\right| \leqq[(r-s) \Delta x]^{1 / 2}\left(2 \Lambda_{p} / a_{0}\right)^{1 / 2} \\
& \leqq K^{\prime \prime}\left(\Lambda_{p}\right)^{1 / 4}
\end{aligned}
$$

for sufficiently large $p$ and the proof follows from Lemma 3 .

Remark. The estimate (4.30) was obtained by Bückner [1] in the selfadjoint case using an elementary device. It would be interesting to know whether or not the discrete eigenvectors display this growth as $M \rightarrow \infty$. In the case of the analytic problem (1.1) it is known (see [15, p. 334]) that the normalized eigenfunctions are uniformly bounded in the supremum norm.

CoRollaRY 2. Let $\left\{V^{p}\right\}_{p=1}^{M}$ be the eigenvectors of $L$ normalized so that $\left\|V^{p}\right\|_{2}=1$, $p=1, \cdots, M$. Then,

$$
\limsup _{M \rightarrow \infty ; \Delta x \rightarrow 0 ;(M+1) \Delta x=1}\left\{\sum_{p=1}^{M} \frac{\left\|V^{p}\right\|_{\infty}}{\Lambda_{p}}\right\}<\infty .
$$

Iroof. This follows immediately from Lemmas 2, 3, and Corollary 1.

Michigan State University

East Lansing, Michigan 48823

1. H. BüCKNeR, "Über Konvergenzsatze, die sich bei der Anwendung eines Differenzenverfahrens auf ein Sturm-Liouvillesches Eigenwertproblem ergeben," Math. Z., v. 51, 1948, pp. 423-465. MR 11, 58.

2. A. Carasso, An Analysis of Numerical Methods for Parabolic Problems Over Long Times, Ph.D. Thesis, Univ. of Wisconsin, Madison, Wis., 1968

3. G. E. Forsythe \& W. R. WAsow, Finite-Difference Methods for Partial Differential Equations, Wiley, New York, 1960. MR 23 \#B3156.

4. F. R. Gantmacher, Matrix Theory, Vol. II, Chelsea, New York, 1964.

5. F. R. Gantmacher \& M. G. KRein, Oscillating Matrices and Kernels and Small Oscillations of Mechanical Systems, Akademie-Verlag, Berlin, 1960; English transl., U.S.A.E.C. 4481, 1961.

6. J. GARY, "Computing eigenvalues of ordinary differential equations by finite differences," Math. Comp., v. 19, 1965, pp. 365̃-379. MR 31 \#4163.

7. A. S. Housenolder, The Theory of Matrices in Numerical Analysis, Blaisdell, New York, 1964. MR $30 \# 5475$.

8. H. B. Keller, "On the accuracy of finite difference approximations to the eigenvalues of differential and integral operators," Numer. Math., v. 7, 1965, pp. 412-419. MR 32 \#6706.

9. S. V. PARTER, "Extreme eigenvalues of Toeplitz forms and applications to elliptic difference equations," Trans. Amer. Math. Soc., v. 99, 1961, pp. 153-192. MR 22 \#11245.

10. S. V. Parter, "On the extreme eigenvalues of Toeplitz matrices," Trans. Amer. Math. Soc., v. 100, 1961, pp. 263-276. MR 25 \#2421.

11. S. V. PARTER, "On the eigenvalues of certain generalizations of Toeplitz matrices," Arch. Rational Mech. Anal., v. 11, 1962, pp. 244-257. MR $26 \# 605$.

12. M. H. Protter \& H. F. Weinberger, Maximum Principles in Differential Equations, Prentice-Hall, Englewood Cliffs, N. J., 1967. MR 36 \#2935.

13. E. A. Coddington \& N. Levinson, Theory of Ordinary Differential Equations, McGrawHill, New York, 1955. MR 16, 1022.

14. E. L. Ince, Ordinary Differential Equations, Dover, New York, 1944. MR 6, 65.

15. R. Courant \& D. Hilbert, Methoden der Mathematischen Physik, Vol. 1, Interscience, New York, 1953. MR 16, 426.

16. D. Greenspan, Introductory Numerical Analysis of Elliptic Boundary Value Problems, Harper \& Row, New York, 1965. MR 31 \#4193.

17. F. W. SindEN, "An oscillation theorem for algebraic eigenvalue problems and its applications," Mitt. Inst. Angew. Math. Zürich, v. 4, 1954, 57 pp. MR 16, 666.

18. R. S. VARGA, Matrix Iterative Analysis, Prentice-Hall, Englewood Cliffs, N. J., 1962. MR. 28 \#1725. 